\title{
A Nonsteroidal Anti-inflammatory Drug, Zaltoprofen, Inhibits the Growth of Extraskeletal Chondrosarcoma Cells by Inducing PPARy, p21, p27, and p53
}

\section{Takashi Higuchi}

Kanazawa University Graduate School of Medical Sciences

Akihiko Takeuchi ( $\square$ a_take@med.kanazawa-u.ac.jp )

Kanazawa University Graduate School of Medical Sciences

\section{Seiichi Munesue}

Kanazawa University Graduate School of Medical Sciences

Norio Yamamoto

Kanazawa University Graduate School of Medical Sciences

Katsuhiro Hayashi

Kanazawa University Graduate School of Medical Sciences

\section{Ai Harashima}

Kanazawa University Graduate School of Medical Sciences

\section{Yasuhiko Yamamoto}

Kanazawa University Graduate School of Medical Sciences

\section{Hiroyuki Tsuchiya}

Kanazawa University Graduate School of Medical Sciences

\section{Research Article}

Keywords: surgical resection, A Nonsteroidal Anti-inflammatory Drug, Zaltoprofen, Extraskeletal Chondrosarcoma Cells

Posted Date: May 5th, 2021

DOl: https://doi.org/10.21203/rs.3.rs-469815/v1

License: (9) (1) This work is licensed under a Creative Commons Attribution 4.0 International License. Read Full License 


\section{Abstract}

There is no effective treatment except surgical resection for extraskeletal myxoid chondrosarcoma (EMC). Peroxisome proliferator-activated receptor gamma (PPARY) is a nuclear receptor and a master transcription factor of adipogenesis-related genes and has been reported as an antitumor target for chondrosarcomas. Herein, we show that a nonsteroidal anti-inflammatory drug, zaltoprofen, induces the expression of PPARY at mRNA and protein levels, following the induction of PPARY-activating factors, such as Krox20,C/EBPB, and C/EBPa, in human extraskeletal chondrosarcoma cells, H-EMC-SS. Zaltoprofen inhibited the proliferation of these cells in vitro. Upregulation of cell cycle checkpoint proteins, p21, p27, and p53, was observed upon treatment of cells with zaltoprofen, which probably resulted in the inhibition of proliferation of H-EMC-SS cells. Zaltoprofen treatment induced tumor necrosis, which could be due to its antiproliferative properties, and was well tolerated in a mouse model of EMC. Our results provide mechanistic insights into the therapeutic effect of zaltoprofen that should promote further studies on the rational use of this drug for effective treatment of EMC.

\section{Introduction}

Extraskeletal myxoid chondrosarcoma (EMC) is a soft tissue sarcoma that arises at various sites, including the extremities ${ }^{1}$. Radiation and chemotherapy being ineffective, wide resection is the only radical treatment available for EMC ${ }^{1}$. Although considered a slow-growing neoplasm, the recurrence and metastatic rates of EMC after radical resection (with values up to $50 \%$ for each) are higher than those for soft-tissue sarcoma ${ }^{1,2}$. Therefore, development of novel treatment strategies is crucial for long-term survival of patients with EMC.

We recently reported that peroxisome proliferator-activated receptor gamma (PPARY), a ligand-activated nuclear receptor that controls the differentiation of adipocytes in normal tissues, could be a therapeutic target for inhibiting cell invasion and matrix metalloproteinase-2 (MMP2) expression in chondrosarcoma cells ${ }^{3}$. In addition, we demonstrated that zaltoprofen, a nonsteroidal anti-inflammatory drug (NSAID), could effectively induce and activate PPARy and elicits antitumor effects in chondrosarcoma cells ${ }^{3}$.

To further understand the therapeutic potential of PPARy-targeted treatment using zaltoprofen, in the present study, we investigated the mechanism of zaltoprofen-mediated induction of PPARY and its antiproliferative effect in vitro and in vivo on human EMC cells.

\section{Materials And Methods}

\section{Reagents.}

Zaltoprofen was purchased from Santa Cruz Biotechnology, Inc. (Dallas, TX, USA) and was dissolved in $10 \%$ dimethyl sulfoxide (DMSO) for in vitro experiments or in $0.2 \%$ carboxymethylcellulose sodium salt (CMC-Na) for in vivo experiments. 


\section{Cell culture.}

The human EMC cell line, H-EMC-SS, was cultured in Dulbecco's modified Eagle's medium (DMEM)/Ham's F-12 (FUJIFILM Wako Pure Chemical Corporation, Osaka, Japan) supplemented with fetal bovine serum $(10 \%)$, penicillin $(100 \mathrm{U} / \mathrm{mL})$, and streptomycin $(100 \mathrm{mg} / \mathrm{mL})$ at $37^{\circ} \mathrm{C}$.

\section{RNA interference.}

PPARY knockdown was conducted using human PPARY short hairpin RNA (shRNA) (SureSilencing shRNA plasmid, Qiagen, Valencia, CA, USA). Attractene transfection reagent (Qiagen) was used to transfect the PPARY shRNA (shPPARY) plasmid or nontargeting shRNA (shNT) control plasmid in H-EMC-SS cells. Neomycin (G418 solution, Roche Diagnostics K.K., Tokyo, Japan) was used for the selection of shRNAtransfected cells, and the knockdown efficiency was confirmed by quantitative reverse transcription polymerase chain reaction (RT-qPCR). Thus, PPARy knockdown cell line (H-EMC-SS ${ }^{\text {shPPARY }}$ ) and nontarget control cell line (H-EMC-SS ${ }^{\text {shNT }}$ ) were established. The shRNA insert sequences were as follows: PPARY shRNA, 5'-CCACGAGATCATTACACAAT-3'; nontargeting shRNA, 5'-GGAATCTCATTCGATGCATAC-3'.

\section{RT-qPCR.}

After treatment with zaltoprofen for $24 \mathrm{~h}, \mathrm{H}$-EMC-SS cells were lysed with TRIzol reagent (Invitrogen, Carlsbad, CA, USA) and total RNA was extracted. The RNA samples (100 ng/sample) were quantified using a spectrophotometer (NanoDrop Lite, Thermo Scientific K.K., Tokyo, Japan), and reverse-transcribed to cDNA on a T100 Thermal Cycler (Bio-Rad Laboratories, Inc., Hercules, CA, USA) using an AffinityScript cDNA Synthesis Kit (Agilent Technologies Inc., Santa Clara, CA, USA). RT-qPCR was performed with $10 \mathrm{ng}$ cDNA per well on a StepOne ${ }^{\text {TM }}$ Real-time PCR System (Applied Biosystems, Foster City, CA, USA) using the QuantiTect SYBR Green PCR Kit (Qiagen). The primers for PPARY, CCAAT/enhancer binding protein $(C / E B P) a, C / E B P \beta, C / E B P \delta$, and Krox20 were purchased from Qiagen (QuantiTect Primer Assay, Hs_PPARG_1_SG, Hs_CEBPA_1_SG, Hs_CEBPB_1_SG, Hs_CEBPD_1_SG, and Hs_EGR2_1_SG). The sequences of primers for glyceraldehyde-3-phosphate dehydrogenase (GAPDH) were as follows: forward, 5'-TGCACCACCAACTGCTTAGC-3'; reverse, 5'-GGCATGGACTGTGGTCATGAG-3'.

\section{Western blot analysis.}

After treatment with various concentrations of zaltoprofen for $24 \mathrm{~h}$, H-EMC-SS cells were lysed in RIPA buffer ( $1 \%$ sodium deoxycholate, $150 \mathrm{mM} \mathrm{NaCl}, 50 \mathrm{mM}$ sodium fluoride, $100 \mu \mathrm{M}$ sodium orthovanadate, $0.1 \%$ sodium dodecyl sulfate [SDS], $1 \%$ Triton $\mathrm{X}-100,10 \mathrm{mM}$ Tris, $\mathrm{pH} 7.2$ ) containing a protease inhibitor cocktail (Sigma-Aldrich Japan, Tokyo, Japan). Total protein ( $100 \mu \mathrm{g} / \mathrm{sample})$ was boiled $\left(2 \mathrm{~min}\right.$ at $\left.95^{\circ} \mathrm{C}\right)$ and resolved by $12.5 \%$ SDS-polyacrylamide gel electrophoresis (PAGE) on an e-PAGEL (ATTO Co., Tokyo, Japan), and transferred onto polyvinylidene fluoride (PVDF) membranes (Merck Millipore Corp., Bedford, 
Germany). After overnight incubation in blocking solution (Odyssey Blocking Buffer PBS, LI-COR Corp., Lincoln, NE, USA), the membranes were incubated with the following antibodies at $4{ }^{\circ} \mathrm{C}$ for $60 \mathrm{~min}$ : antiPPARy antibody (Santa Cruz), anti-p21 (Cell Signaling Technology, Danvers, MA, USA), anti-p27 (Santa Cruz), anti-p53 (Santa Cruz), and anti-GAPDH (Cell Signaling Technology). The membranes were washed three times with phosphate-buffered saline containing 10\% Tween 20 (T-PBS) and incubated with the secondary antibody (IRDye 680RD goat anti-IgG secondary antibody, LI-COR) for $30 \mathrm{~min}$. After washing three times with T-PBS, the bands were visualized and scanned with an Odyssey Infrared Imaging system (LI-COR).

\section{Water-soluble tetrazolium salt-8 (WST-8) assay.}

The growth of cells at designated time points after zaltoprofen treatment was determined using a Cell Counting Kit-8 (Dojindo Laboratory, Kumamoto, Japan). After H-EMC-SS cells $\left(5 \times 10^{3}\right.$ cells/well) were treated with the indicated concentrations of zaltoprofen, the absorbance at $450 \mathrm{~nm}$ was measured using an iMark Microplate Absorbance Reader (Bio-Rad Laboratories).

\section{5-ethynyl-2'-deoxyuridine (EdU) proliferation assay.}

After incubating the H-EMC-SS ${ }^{\text {ShPPARY }}$ and H-EMC-SS ${ }^{\text {ShNT }}$ cells on slide chambers for 24 h, they were further incubated with $1 \%$ DMSO or $400 \mu \mathrm{mol} / \mathrm{L}$ zaltoprofen for $24 \mathrm{~h}$. The cells were then treated with 10 $\mathrm{mmol} / \mathrm{L}$ EdU stock solution (Click-iT ${ }^{\circledR}$ EdU Alexa Fluor ${ }^{\circledR} 555$ Imaging Kit, Invitrogen) for 60 min, fixed with $4 \%$ paraformaldehyde (Wako), washed twice with $3 \%$ bovine serum albumin (BSA), permeabilized with $0.5 \%$ Triton X-100, incubated with the Click-iT reaction buffer (Click-iT ${ }^{\circledR}$ EdU Alexa Fluor ${ }^{\circledR} 555$ Imaging Kit) for $30 \mathrm{~min}$, and washed once with BSA. The nuclei of the cells were stained with Hoechst 33342 (NucBlue Live ReadyProbes Reagent, Invitrogen). Cells positive for EdU-stained nuclei were visualized with a fluorescence microscope (BZ-9000, Keyence Co., Osaka, Japan). EdU-positive and Hoechst 33342positive cells were counted using the ImageJ software (National Institute of Health, USA).

\section{Animal experiments.}

Four-week-old BALB/c female nude mice (Charles River Laboratories Japan Inc., Kanagawa, Japan) were adapted to the conditions for a week before the experiment.

H-EMC-SS cells suspended in Matrigel (BD Japan, Tokyo, Japan) $\left(4 \times 10^{6} / 100 \mu \mathrm{L}\right)$ were subcutaneously implanted on the flanks of mice. The mice were randomly divided into zaltoprofen $(30 \mathrm{mg} / \mathrm{kg}$, oral gavage, twice a day, 42 days)-treated $(n=6)$ and vehicle $(0.2 \% \mathrm{CMC}-\mathrm{Na}, 10 \mathrm{~mL} / \mathrm{kg}$, oral gavage twice a day for 42 days)-treated $(n=6)$ groups. Tumor diameter and body weight of mice were measured once a week using digital calipers and digital scales, respectively. Tumor volume $\left(\mathrm{mm}^{3}\right)$ was calculated using the 
following formula: Tumor volume $\left(\mathrm{mm}^{3}\right)=$ largest diameter $(\mathrm{mm}) \times$ smallest diameter $(\mathrm{mm}) \times$ smallest diameter $(\mathrm{mm}) \times 0.5$.

After 42 days of treatment, the mice were humanely sacrificed and scanned by computed tomography (CT), and tumors were resected for histopathological evaluation. The resected tumors were fixed in $10 \%$ formalin and embedded in paraffin. Tumor sections were deparaffinized and rehydrated in xylene and ethanol, and stained with hematoxylin and eosin (HE) or immunostained with antibodies against Ki-67 (Abcam, Cambridge, UK) in combination with diaminobenzidine (DAB, Dako Japan Inc., Kyoto, Japan). Terminal deoxynucleotidyl transferase dUTP nick-end labeling (TUNEL) assay using a cell death kit (Roche) was performed according to the manufacturer's instructions. TUNEL-stained area was measured and Ki-67-positive cells were counted using the Image $\mathrm{J}$ software. Kidney, intestine, and blood serum samples were collected from mice and evaluated pathologically and biochemically by an outsourced inspection facility (Safety Research Institute for Chemical Compounds Co., Ltd., Sapporo, Japan) for evaluation of the side effects.

\section{Statistical analysis.}

Easy R (Saitama Medical Center, Jichi Medical University, Saitama, Japan), a modified version of R commander (The R Foundation for Statistical Computing, Vienna, Austria), which includes statistical functions for biostatistics, was used for all statistical analyses ${ }^{4}$. Data were compared using Student's $t$ test or one-way analysis of variance (ANOVA) with Tukey's post-hoc test. All data are expressed as mean \pm standard error of the mean (SEM). All $p$-values were two-sided.

\section{Ethics approval}

All research was performed in accordance with relevant guidelines and regulations. The study protocol was approved by Kanazawa University Advanced Science Research Centre (Kanazawa, Japan). All procedures for animal studies were performed in accordance with the Japanese national guidelines, and the protocol was approved by the Institute for Experimental Animals, Kanazawa University Advanced Science Research Center. The authors complied with the ARRIVE guidelines.

\section{Results}

\section{Induction of PPARy by zaltoprofen.}

Zaltoprofen treatment upregulated the mRNA and protein levels of PPARY in H-EMC-SS cells as evidenced by qRT-PCR and western blot analysis, respectively, demonstrating that zaltoprofen can induce PPARY in EMC (Fig. 1a and b). Next, we investigated the induction of Krox20 and C/EBPs, which regulate PPARY in the normal fat tissue, in zaltoprofen-treated H-EMC-SS cells. Krox20 was significantly upregulated from 3 to $24 \mathrm{~h}$ after the initiation of treatment, followed by a significant upregulation of $C / E B P \beta$ starting $12 \mathrm{~h}$ 
and of $C / E B P a$ at $24 \mathrm{~h}$, demonstrating the signal pathway of PPARy induction by zaltoprofen in EMC (Fig. 1C).

\section{Inhibition of cell proliferation by zaltoprofen.}

The inhibitory effect of zaltoprofen on the proliferation of $\mathrm{H}$-EMC-SS cells in vitro was examined by the WST-8 and EdU assays. After treatment of H-EMC-SS cells with zaltoprofen $(0-800 \mu \mathrm{mol} / \mathrm{L})$ for $96 \mathrm{~h}$, their growth was significantly reduced in a dose- and time-dependent manner (Fig. 2a). To investigate the PPARY-dependency, the cell growth was analyzed after downregulation of PPARy using an shRNA. The growth of H-EMC-SS ${ }^{\text {shPPARY }}$ cells was significantly increased compared to that of H-EMC-SS ${ }^{\text {shNT }}$ cells with and without $400 \mu \mathrm{mol} / \mathrm{L}$ zaltoprofen treatment $(p<0.001$, Fig. $2 b)$. However, zaltoprofen treatment also significantly inhibited the growth of H-EMC-SS ${ }^{\text {shPPARY }}$ cells $(p<0.001)$, suggesting that zaltoprofen also exerts PPARy-independent effects on the growth of cells.

The EdU-positive cell ratio (EdU-incorporated cells/Hoechst 33342-positive nuclei) was calculated, and the PPARY-dependency of cell proliferation was confirmed by silencing of PPARY in the cells (Fig. 3). Zaltoprofen significantly and dose-dependently decreased the EdU-positive cell ratio in H-EMC-SS ${ }^{\text {shNT }}$ cells. The EdU-positive cell ratio in H-EMC-SS ${ }^{\text {shPPARy }}$ cells was significantly higher than that in H-EMC$\mathrm{SS}^{\text {shNT }}$ cells. However, zaltoprofen treatment significantly reduced the EdU-positive ratio of H-EMCSS ${ }^{\text {shPPARY }}$ cells in a dose-dependent manner. These data suggest that proliferation of EMC cells is mediated by PPARY and the antiproliferation effect of zaltoprofen on EMC cells is both PPARY-dependent and -independent.

Expression of cell cycle checkpoint proteins, namely p21, p27, and p53, which regulate abnormal cycling of cancer cells, was measured by western blot analysis in both H-EMC-SS ${ }^{\text {shNT }}$ and H-EMC-SS ShPPARY cells (Fig. 4). Zaltoprofen treatment induced the expression of p21, p27, and p53 in H-EMC-SS ${ }^{\text {shNT }}$ cells but not in H-EMC-SS ${ }^{\text {shPPARY }}$ cells, demonstrating that zaltoprofen could induce cell-cycle checkpoint proteins in a PPARY-dependent manner in EMC cells.

\section{Inhibitory effects of zaltoprofen on xenograft tumors in mice.}

The effect of zaltoprofen on xenograft tumors and its safety were tested using xenograft mouse models subcutaneously implanted with H-EMC-SS cells. We checked the effect of $400 \mu \mathrm{mol} / \mathrm{L}$ of zaltoprofen, which is an effective concentration in vitro, and equivalent to $30 \mathrm{mg} / \mathrm{kg}$ in vivo. Zaltoprofen $(30 \mathrm{mg} / \mathrm{kg})$ treatment significantly inhibited the tumor growth at all time points compared with that in the vehicle group ( $p=0.032$, Fig. 5a). CT images of the zaltoprofen-treated tumor at the treatment end point showed decreased tumor size and increased calcification inside the tumor (Fig. 5b and c). Histopathological analyses were conducted on excised tumors. HE-stained sections showed that tumors in the vehicle 
group consisted of viable highly dense tumor cells, whereas zaltoprofen-treated tumors had scattered scar areas with infiltration of inflammatory cells (Fig. $5 \mathrm{~d}$ and e). TUNEL-positive tumors in zaltoprofentreated group were significantly larger than those in the vehicle group $(p=0.024$, Fig. 6a-c), demonstrating that zaltoprofen induced necrosis of tumors in the H-EMC-SS xenograft models. In contrast, tumors in the zaltoprofen-treated group had significantly lower Ki-67 positive cells compared with those in the vehicle group ( $p<0.001$, Fig. $6 d-f)$, demonstrating that zaltoprofen inhibited the cell cycle in the xenograft tumors. The body weights in both vehicle- and zaltoprofen-treated groups post-administration were higher than the pre-administration weights $(p=0.005$ and $p=0.014$, respectively; Fig. 7. Generally, long-term administration of NSAIDs can cause kidney injury, although there was no abnormal renal function, as evident from the absence of abnormalities in the levels of blood urea nitrogen and serum creatinine, or pathological abnormalities in renal autopsy in both the groups. In addition, no hepatotoxicity was detected in the analysis of serum total bilirubin in either of the groups. There were no other observable side effects or deaths in either of the mouse groups.

\section{Discussion}

We found that zaltoprofen induced the expression of PPARY in human EMC cells, H-EMC-SS, in a dosedependent manner by inducing KROX20,C/EBP $\beta$, and $C / E B P a$ in that order (Fig. 1). Zaltoprofen treatment induced the expression of cell cycle checkpoint proteins, p21, p27, and p53, and significantly inhibited the proliferation of H-EMC-SS cells (Fig. 2-4). The knockdown of PPARy did not completely cancel the antitumor effect of zaltoprofen (Fig. 2b and Fig. 3), suggesting that zaltoprofen affects cell proliferation in both PPARY-dependent and -independent manner. The antiproliferative effects of zaltoprofen on $\mathrm{H}$ EMC-SS cells were demonstrated in vivo (Fig. 5-7).

The role of PPARY in malignant tumor biology has been studied, and PPARy ligands are known to elicit antitumor effects in cancer cells ${ }^{3,5-7}$. Several drugs, including the antidiabetic and antihypertensive ones, and NSAIDs, are potential PPARy ligands. Among NSAIDs, indometacin, oxaprozin, diclofenac, and zaltoprofen are reported to be potential PPARY ligands ${ }^{8-10}$. In the present study, we demonstrate, for the first time, that zaltoprofen induces PPARy, and elicits antitumor effects against musculoskeletal tumors, both in vitro and in vivo.

C/EBPs form a family of six homologous transcription factors that are involved in regulating cellular proliferation and differentiation, metabolism, and immunity in various organs ${ }^{11,12}$. C/EBP $\beta$ and C/EBP are induced early during adipogenesis and are involved in the induction of PPARY ${ }^{13,14}$. C/EBPa is induced at a later stage, which is almost the same stage at which PPAR is induced, and is activated in mature adipocytes ${ }^{15,16}$. C/EBPa promotes adipogenesis both with and without PPARY induction ${ }^{15,16}$. Krox20, also referred to as early growth response protein 2 (EGR2), a transcription regulatory factor, is an upstream regulator of C/EBPs and one of the earliest factors to be induced during adipogenesis ${ }^{17,18}$. Herein, we demonstrate that, in H-EMC-SS cells, zaltoprofen induces Krox20 at the earliest stage, followed 
by $C / E B P \beta$ and $C / E B P a$, but not $C / E B P \delta$. This pathway could be one of the mechanisms for the induction of PPARY by zaltoprofen in H-EMC-SS cells, but it needs to be confirmed for the other malignant cells.

The cyclin-dependent kinase (CDK) inhibitors, p21 and p27, are essential tumor suppressor proteins that play significant roles in the inhibition of the growth of cancer cells ${ }^{19}$. It has been reported that activation of PPARy induces cell cycle arrest through the upregulation of p21 and p27 in various cancer cells, such as hepatic cell carcinoma ${ }^{20-22}$, melanoma ${ }^{23}$, and pancreatic carcinoma ${ }^{24}$. The tumor suppressor protein, p53, also regulates the cell cycle by repairing DNA, inducing apoptosis, and inducing p21 and p27; thus, p21, p27, and p53 interact in complex manner to suppress the cell cycle ${ }^{25}$. In the present study, p21, p27, and p53 were induced in H-EMC-SS cells upon zaltoprofen treatment in a PPARY-dependent manner and inhibited the proliferation of cells. Whether zaltoprofen induces p21 or p27 in a p53dependent or -independent manner should be examined in future studies. In addition, the PPARYindependent antitumor effects of zaltoprofen, as evident in the WST-8 and EdU assays in the present study, would require further confirmation in future studies.

In the present study, we have demonstrated the in vivo antiproliferative effect of zaltoprofen on musculoskeletal tumor cells for the first time. The downregulation of Ki-67, a proliferation marker expressed during the active cell-cycle phases, in zaltoprofen-treated tumors indicates the inhibition of cell cycle in vivo by zaltoprofen, which corroborates with the in vitro results. There were more TUNEL-stained cells in zaltoprofen-treated tumors, indicating that zaltoprofen could induce necrosis of tumor cells. The good tolerability of zaltoprofen, which was revealed by the absence of major complications, including body weight loss, nephrotoxicity, and hepatotoxicity, should be useful in carrying out further analysis on the efficacy of zaltoprofen against musculoskeletal tumors for potential clinical application.

In conclusion, we demonstrate that zaltoprofen induces Krox20,C/EBPB, C/EBPa, and PPARY, and subsequently p21, p27, and p53 in EMC cells, and contributes to the inhibition of cell proliferation. The antiproliferative effect of zaltoprofen is also demonstrated in vivo with good tolerability. These results suggest that zaltoprofen could be a novel and effective treatment for EMC.

\section{Declarations}

\section{Acknowledgements}

This work was supported by JSPS KAKENHI Grant Number JP17K16682.

\section{Author contributions}

T.H. designed research, conducted experiments, analyzed and interpreted data, and wrote the manuscript. A.T. conceived and designed research, and approved the manuscript. S.M. and A.H. supported experiments, interpreted data, and approved the manuscript. N.Y. and K.H. supported experiments and 
approved the manuscript. Y.Y. interpreted data, and reviewed and approved the manuscript. H.T. conceived research and approved the manuscript.

\section{Competing interests}

The authors declare no competing interests.

\section{References}

1. Paioli, A. et al. Extraskeletal Myxoid Chondrosarcoma with Molecularly Confirmed Diagnosis: A Multicenter Retrospective Study Within the Italian Sarcoma Group. Ann. Surg. Oncol. 28, 1142-1150 (2021).

2. Kawaguchi, S. et al. Extraskeletal myxoid chondrosarcoma: a Multi-Institutional Study of 42 Cases in Japan. Cancer. 97, 1285-1292 (2003).

3. Higuchi, T. et al. Anti-tumor effects of a nonsteroidal anti-inflammatory drug zaltoprofen on chondrosarcoma via activating peroxisome proliferator-activated receptor gamma and suppressing matrix metalloproteinase-2 expression. Cancer Med. 7, 1944-1954 (2018).

4. Kanda, Y. Investigation of the freely available easy-to-use software 'EZR' for medical statistics. Bone Marrow Transplant. 48, 452-458 (2013).

5. Augimeri, G. et al. Natural and Synthetic PPARy Ligands in Tumor Microenvironment: A New Potential Strategy against Breast Cancer. Int. J. Mol. Sci. 21, 9721 (2020).

6. Ishay-Ronen, D. \& Christofori, G. Targeting Cancer Cell Metastasis by Converting Cancer Cells into Fat. Cancer Res. 79, 5471-5475 (2019).

7. Vallée, A., Lecarpentier, Y. \& Vallée, J. N. Targeting the Canonical WNT/B-Catenin Pathway in Cancer Treatment Using Non-Steroidal Anti-Inflammatory Drugs. Cells. 8, 726 (2019).

8. Carvalho, M. V., Gonçalves-de-Albuquerque, C. F. \& Silva, A. R. PPAR Gamma: From Definition to Molecular Targets and Therapy of Lung Diseases. Int. J. Mol. Sci. 22, 805 (2021).

9. Matheson, J. \& Le Foll, B. Therapeutic Potential of Peroxisome Proliferator-Activated Receptor (PPAR) Agonists in Substance Use Disorders: A Synthesis of Preclinical and Human Evidence. Cells. 9, 1196 (2020).

10. Yamazaki, R., Kusunoki, N., Matsuzaki, T., Hashimoto, S. \& Kawai, S. Nonsteroidal anti-inflammatory drugs induce apoptosis in association with activation of peroxisome proliferator-activated receptor gamma in rheumatoid synovial cells. J. Pharmacol. Exp. Ther. 302, 18-25 (2002).

11. Tolomeo, M. \& Grimaudo, S. The "Janus" Role of C/EBPs Family Members in Cancer Progression. Int. J. Mol. Sci. 21, 4308 (2020).

12. Wang, W., Xia, X., Mao, L. \& Wang, S. The CCAAT/Enhancer-Binding Protein Family: Its Roles in MDSC Expansion and Function. Front. Immunol. 10, 1804 (2019). 
13. Farmer, S. R. Regulation of PPARgamma activity during adipogenesis. Int. J. Obes. (Lond). 29 (Suppl 1), S13-16 (2005).

14. Lee, J. E., Cho, Y. W., Deng, C. X. \& Ge, K. MLL3/MLL4-Associated PAGR1 Regulates Adipogenesis by Controlling Induction of C/EBP $\beta$ and C/EBPS. Mol. Cell. Biol. 40, e00209-20 (2020).

15. Madsen, M. S., Siersbæk, R., Boergesen, M., Nielsen, R. \& Mandrup, S. Peroxisome proliferatoractivated receptor $\mathrm{Y}$ and $\mathrm{C} / \mathrm{EBPa}$ synergistically activate key metabolic adipocyte genes by assisted loading. Mol. Cell. Biol. 34, 939-954 (2014).

16. Rosen, E. D. et al. C/EBPalpha induces adipogenesis through PPARgamma: a unified pathway. Genes Dev. 16, 22-26 (2002).

17. Chen, Z., Torrens, J. I., Anand, A., Spiegelman, B. M. \& Friedman, J. M. Krox20 stimulates adipogenesis via C/EBPbeta-dependent and -independent mechanisms. Cell Metab. 1, 93-106 (2005).

18. Park, Y. K. et al. Distinct Roles of Transcription Factors KLF4, Krox20, and Peroxisome ProliferatorActivated Receptor $\mathrm{Y}$ in Adipogenesis. Mol. Cell. Biol. 37, e00554-16 (2017).

19. Abukhdeir, A. M. \& Park, B. H. P21 and p27: roles in carcinogenesis and drug resistance. Expert Rev. Mol. Med. 10, e19 (2008).

20. Motomura, W. et al. Growth arrest by troglitazone is mediated by p27Kip1 accumulation, which results from dual inhibition of proteasome activity and Skp2 expression in human hepatocellular carcinoma cells. Int. J. Cancer. 108, 41-46 (2004).

21. Rumi, M. A. et al. Peroxisome proliferator-activated receptor gamma ligand-induced growth inhibition of human hepatocellular carcinoma. Br. J. Cancer. 84, 1640-1647 (2001).

22. Cheung, K. F. et al. CITED2 is a novel direct effector of peroxisome proliferator-activated receptor $y$ in suppressing hepatocellular carcinoma cell growth. Cancer. 119, 1217-1226 (2013).

23. Flori, E. et al. The a-melanocyte stimulating hormone/peroxisome proliferator activated receptor- $\gamma$ pathway down-regulates proliferation in melanoma cell lines. J. Exp. Clin. Cancer Res. 36, 142 (2017).

24. Vitale, G. et al. The PPAR-y agonist troglitazone antagonizes survival pathways induced by STAT-3 in recombinant interferon- $\beta$ treated pancreatic cancer cells. Biotechnol. Adv. 30, 169-184 (2012).

25. Philipp-Staheli, J. et al. Distinct roles for p53, p27Kip1, and p21Cip1 during tumor development. Oncogene. 23, 905-913 (2004).

\section{Figures}


Figure 1 (a)

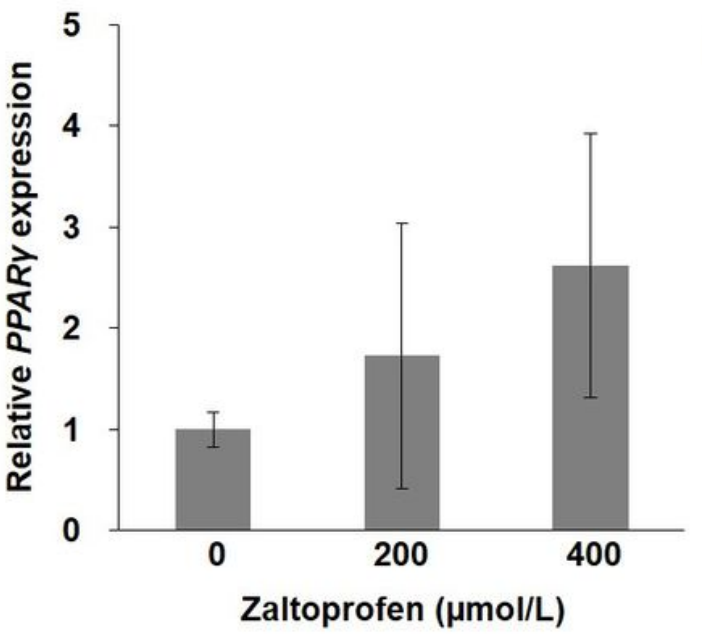

(b)

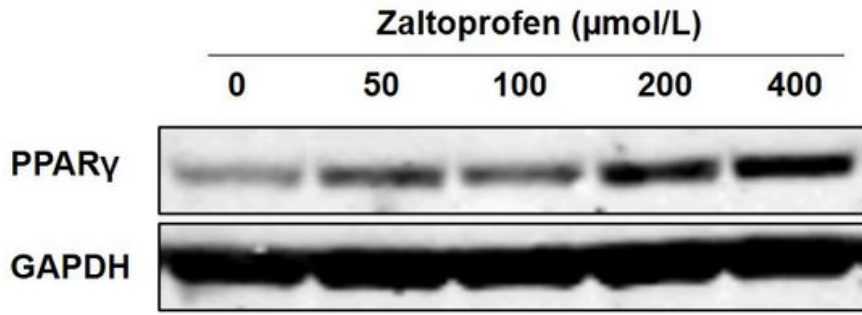

(c)

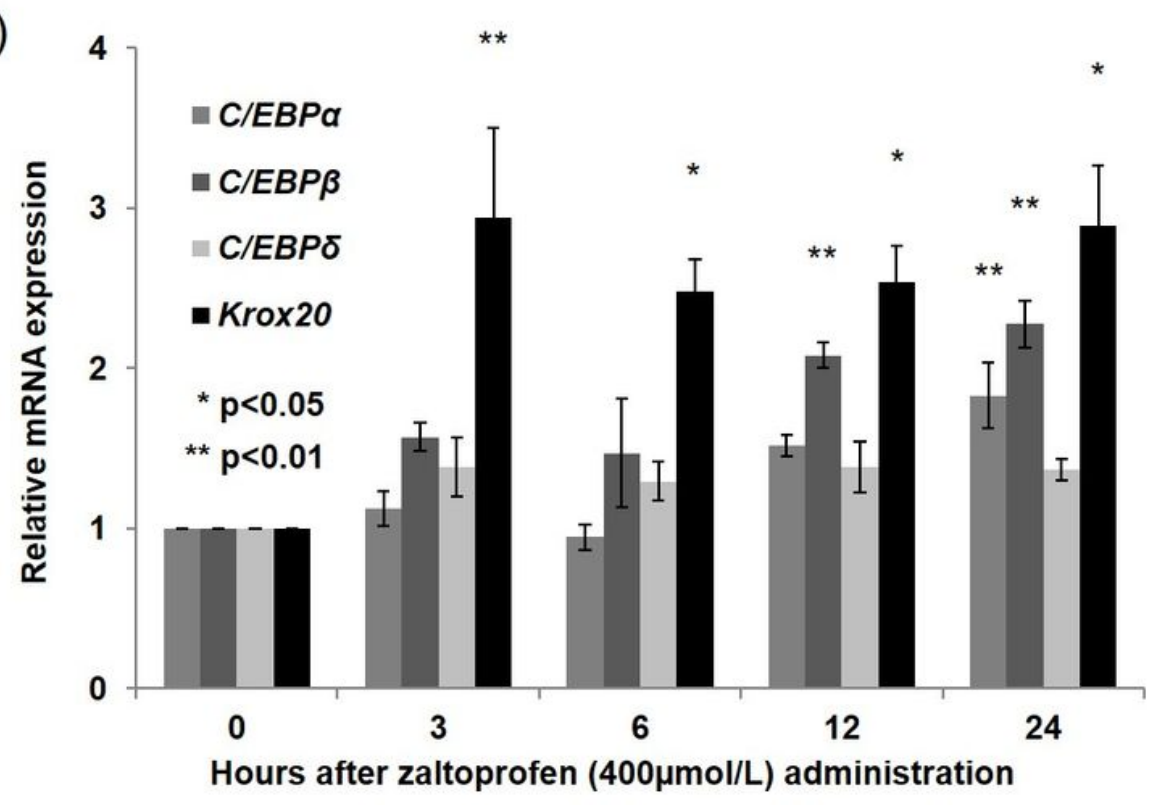

Figure 1

Effect of zaltoprofen on the expression of PPARY in H-EMC-SS cells at the mRNA (a) and protein (b) levels, as determined by qRT-PCR and western blot analysis, respectively. GAPDH was used as a housekeeping factor. (c) Effect of zaltoprofen on the expression of C/EBPa, C/EBPB, C/EBPS, and Krox20 mRNAs in H-EMC-SS cells, as determined by qRT-PCR. ${ }^{\star} p<0.05,{ }^{\star *} p<0.01$. Error bars: \pm SEM. 
(a)

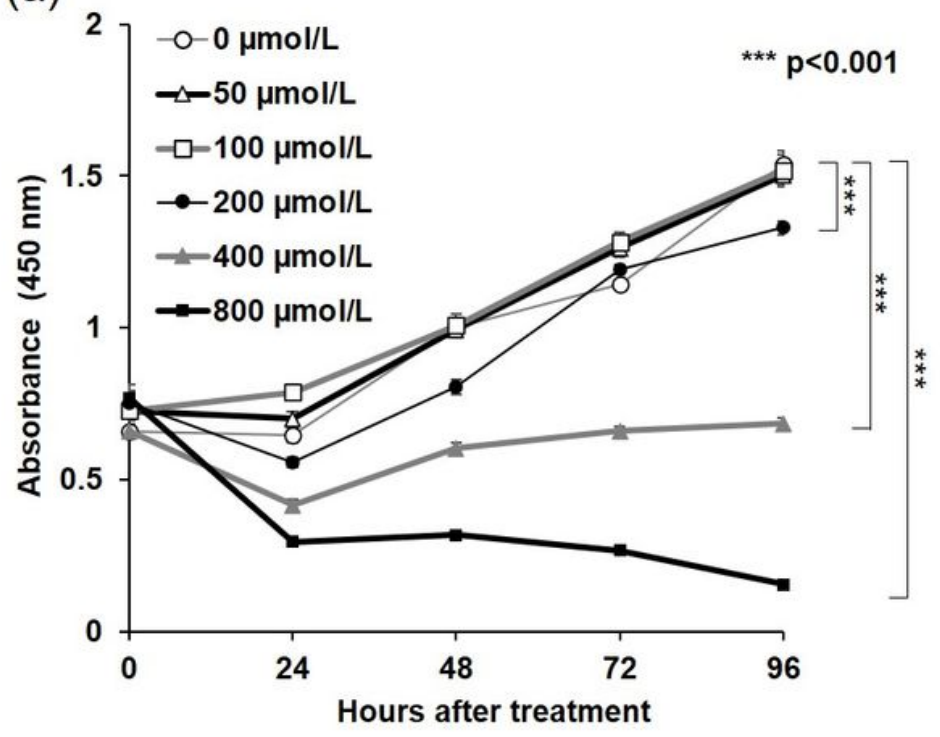

(b)

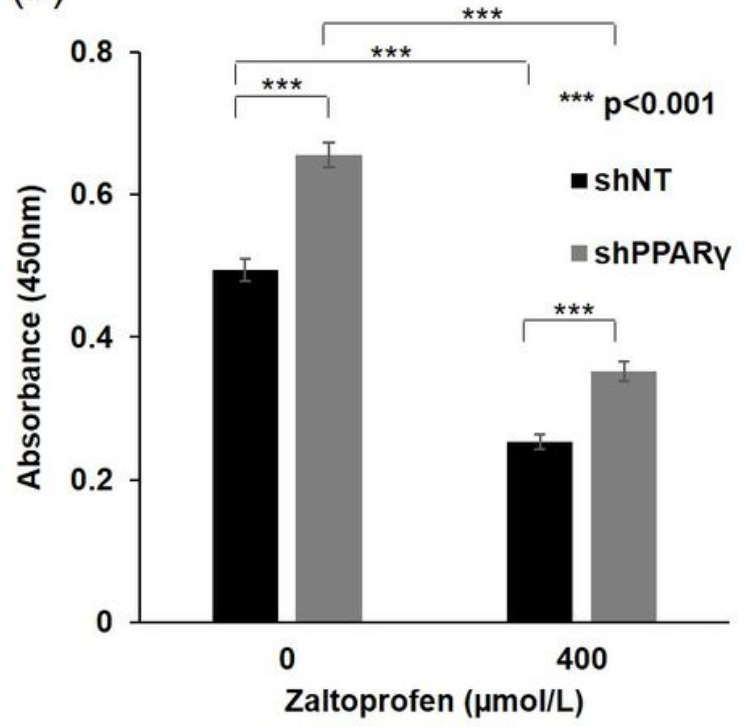

Figure 2

(a) Effects of zaltoprofen on the viability of H-EMC-SS cells as detected by the WST-8 assay. The cells were treated with various concentrations of zaltoprofen and absorbance was measured at each time point. (b) PPARy dependency of the effect of zaltoprofen on cell viability. H-EMC-SSshNT and H-EMCSSshPPARy cells were treated with or without zaltoprofen for $96 \mathrm{~h}$ before the WST-8 assay. ${ }^{* \star *} \mathrm{p}<0.001$. Error bars: \pm SEM.

Figure 3

(a)

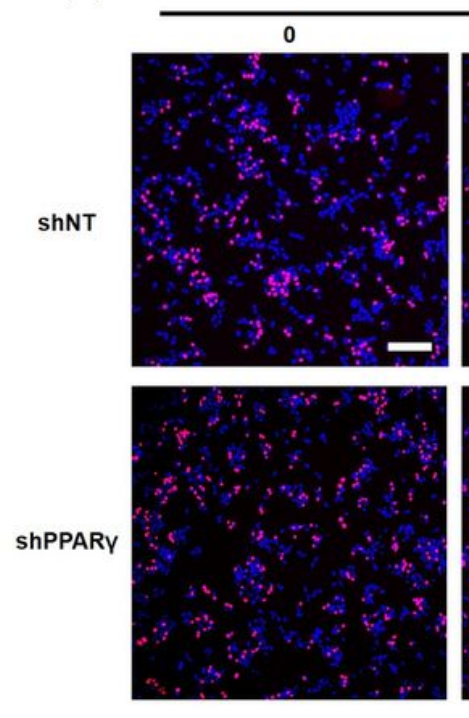

Zaltoprofen ( $\mu \mathrm{mol} / \mathrm{L}$ )
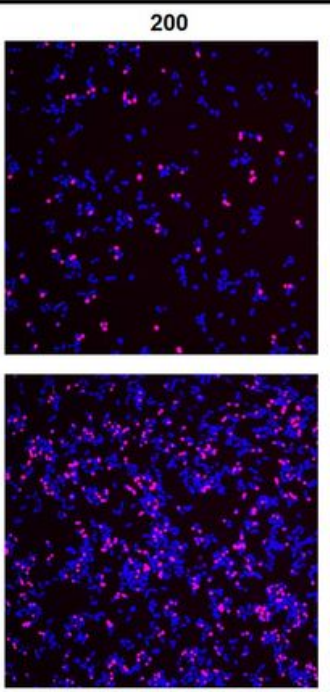
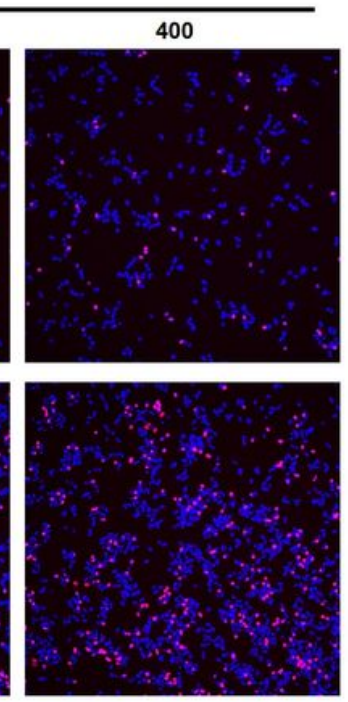

(b)

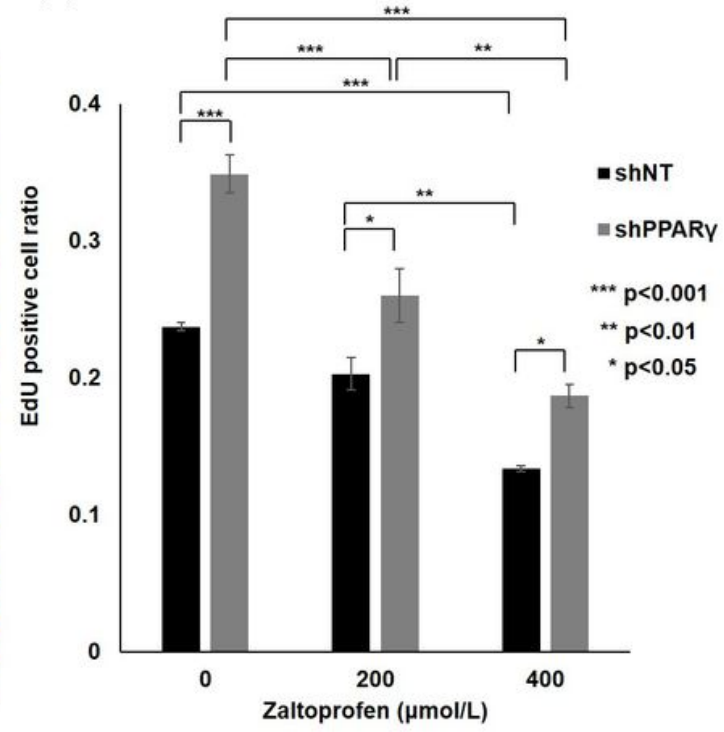

Figure 3 
(a) Representative photographs of H-EMC-SSshNT and H-EMC-SSshPPARY cells with/without the zaltoprofen treatment for $24 \mathrm{~h}$ in EdU proliferation assay. Scale bar $=100 \mu \mathrm{m}$. (b) The ratio of EdUpositive cells (the number of red nuclei [EdU-positive] per the number of blue nuclei [Hoechst $33342-$ positive]). ${ }^{*} p<0.05,{ }^{* *} p<0.01,{ }^{* \star *} p<0.001$. Error bars: \pm SEM.

\section{Figure 4}
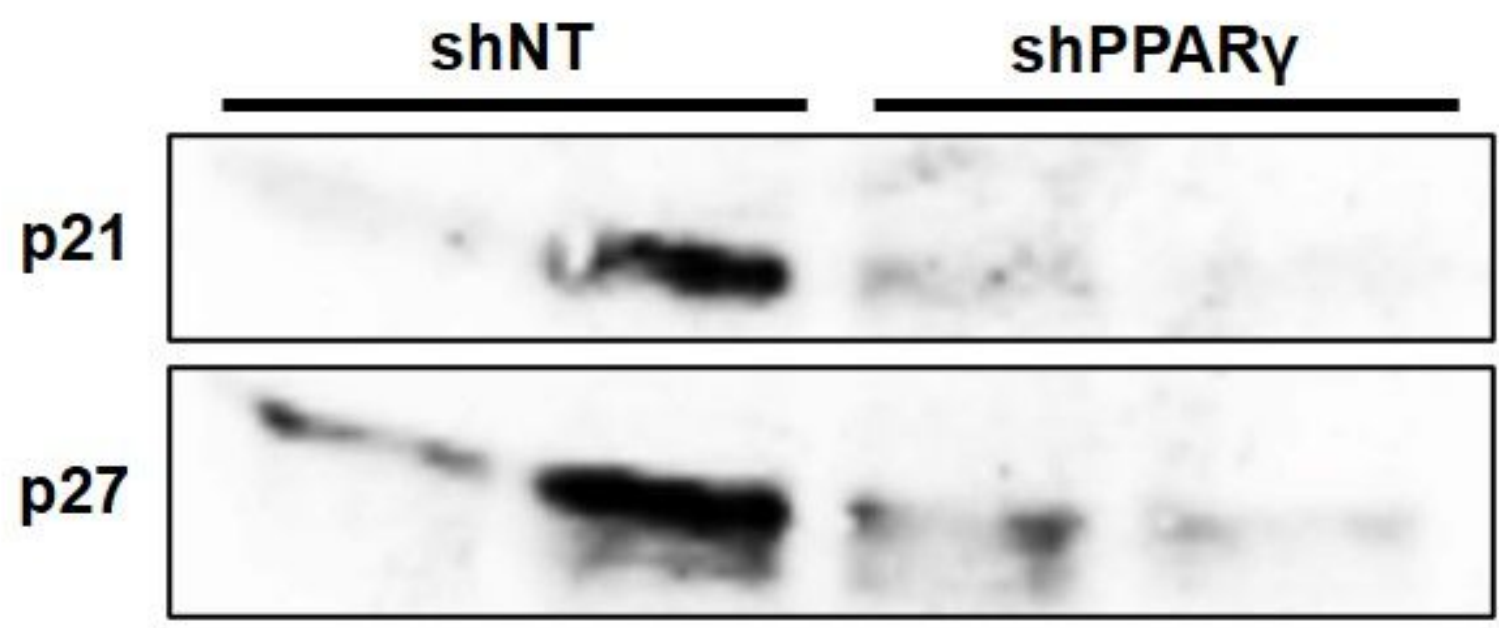

\section{GAPDH}

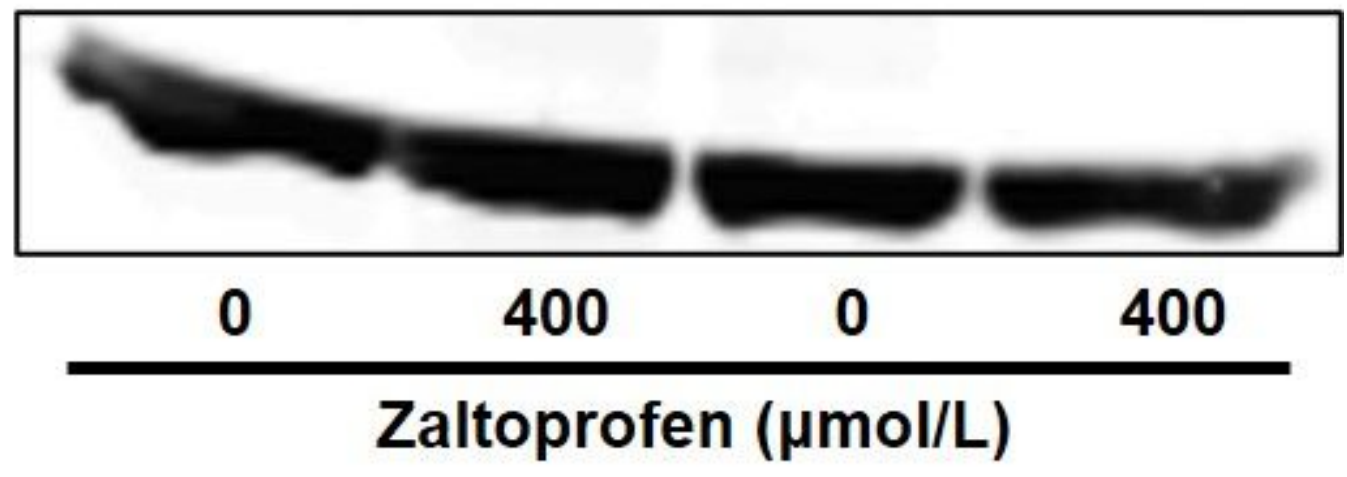

Figure 4

Effects of zaltoprofen on the expression of p21, p27, and p53 in H-EMC-SSshNT and H-EMC-SSshPPARY cells, as detected by western blot analysis. GAPDH was used as a housekeeping protein. 
Figure 5
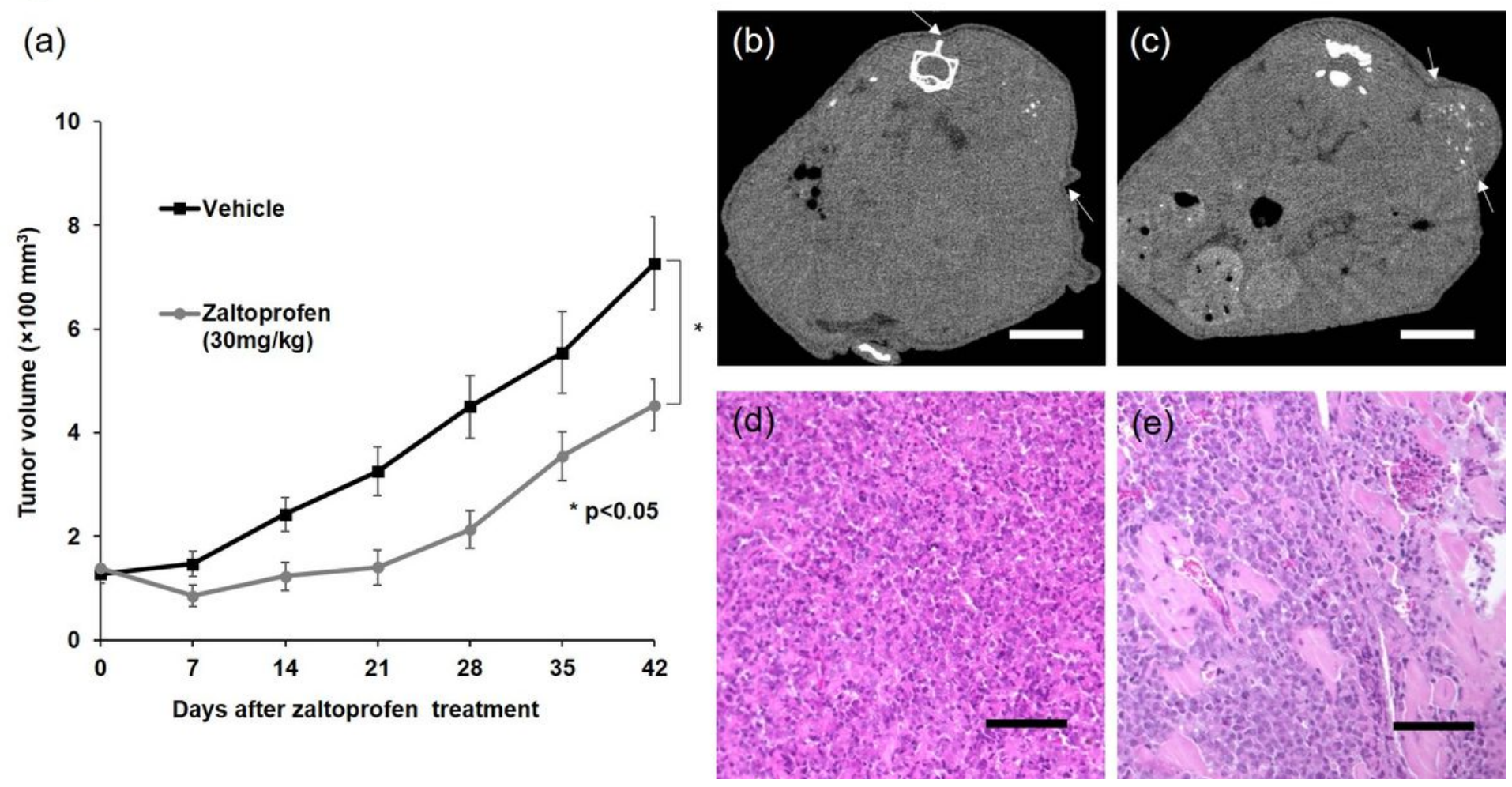

Figure 5

(a) Tumor growth curves for the extraskeletal myxoid chondrosarcoma (EMC) xenograft model with or without zaltoprofen treatment. Line graphs indicate the tumor volume at each time point after the initiation of treatment. (b, c) Representative computed tomography (CT) images of mouse models on the final day. Arrows show the boundary of the tumors. (b) Vehicle treated group. (c) Zaltoprofen treated group. Scale bar $=10 \mathrm{~mm}$. ( (d, e) Hematoxylin and eosin (HE) staining of the tumors. (d) Vehicle treated group. (e) Zaltoprofen treated group. Scale bar $=100 \mu \mathrm{m}$. ${ }^{\star} \mathrm{p}<0.05$. Error bars: \pm SEM. 
Figure 6

(a)

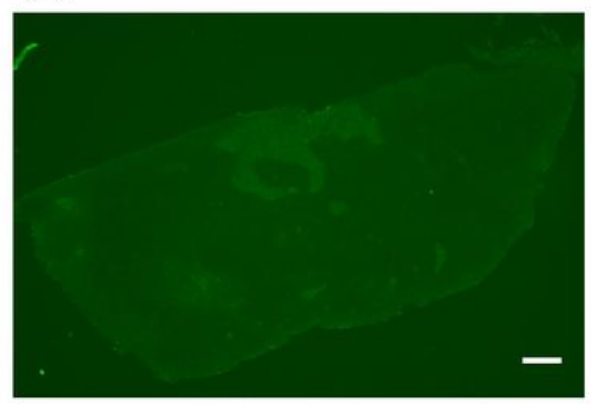

(d)

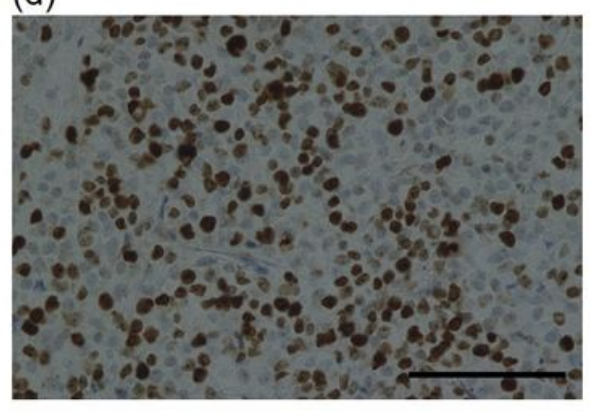

(b)

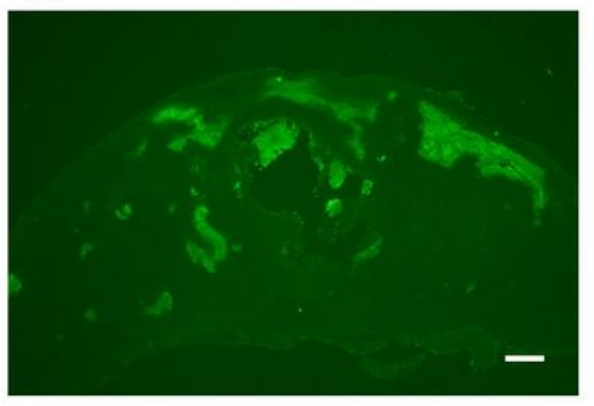

(e)

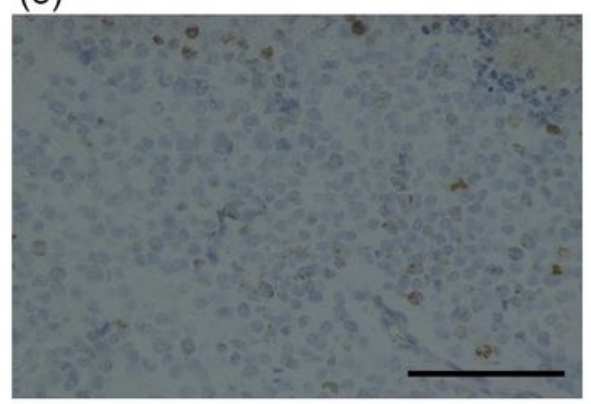

(c)

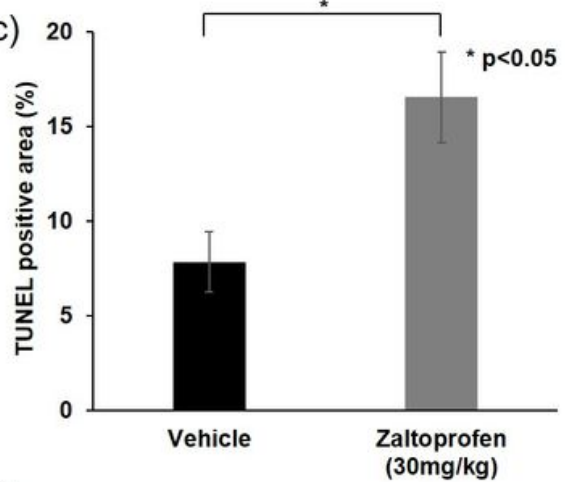

(f)

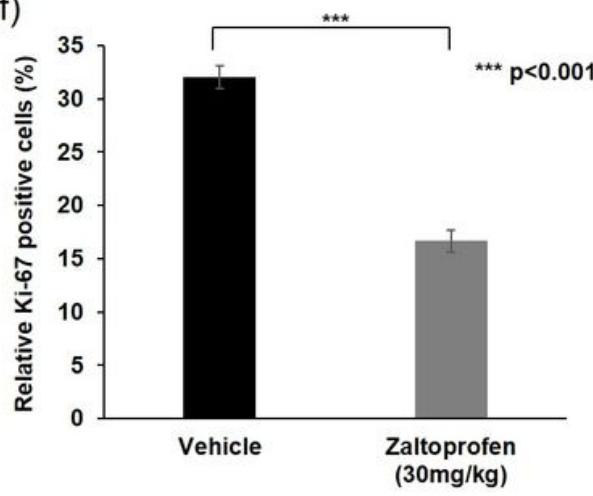

Figure 6

(a, b) Representative photographs of TUNEL-stained tumors. (a) Vehicle treated group. (b) Zaltoprofen treated group. Scale bar $=100 \mu \mathrm{m}$ (c) Bar graphs indicate the average TUNEL-positive area (percentage of fluorescent area in the tumor region). (d, e) Representative photographs of tumors immunohistochemically stained for Ki-67. (d) Vehicle treated group. (e) Zaltoprofen treated group. Scale bar $=100 \mu \mathrm{m}$. (f) Bar graphs indicate the percentage of Ki-67-positive cells. ${ }^{*} p<0.05,{ }^{*} * \mathrm{p}<0.001$. Error bars: \pm SEM. 
Figure 7

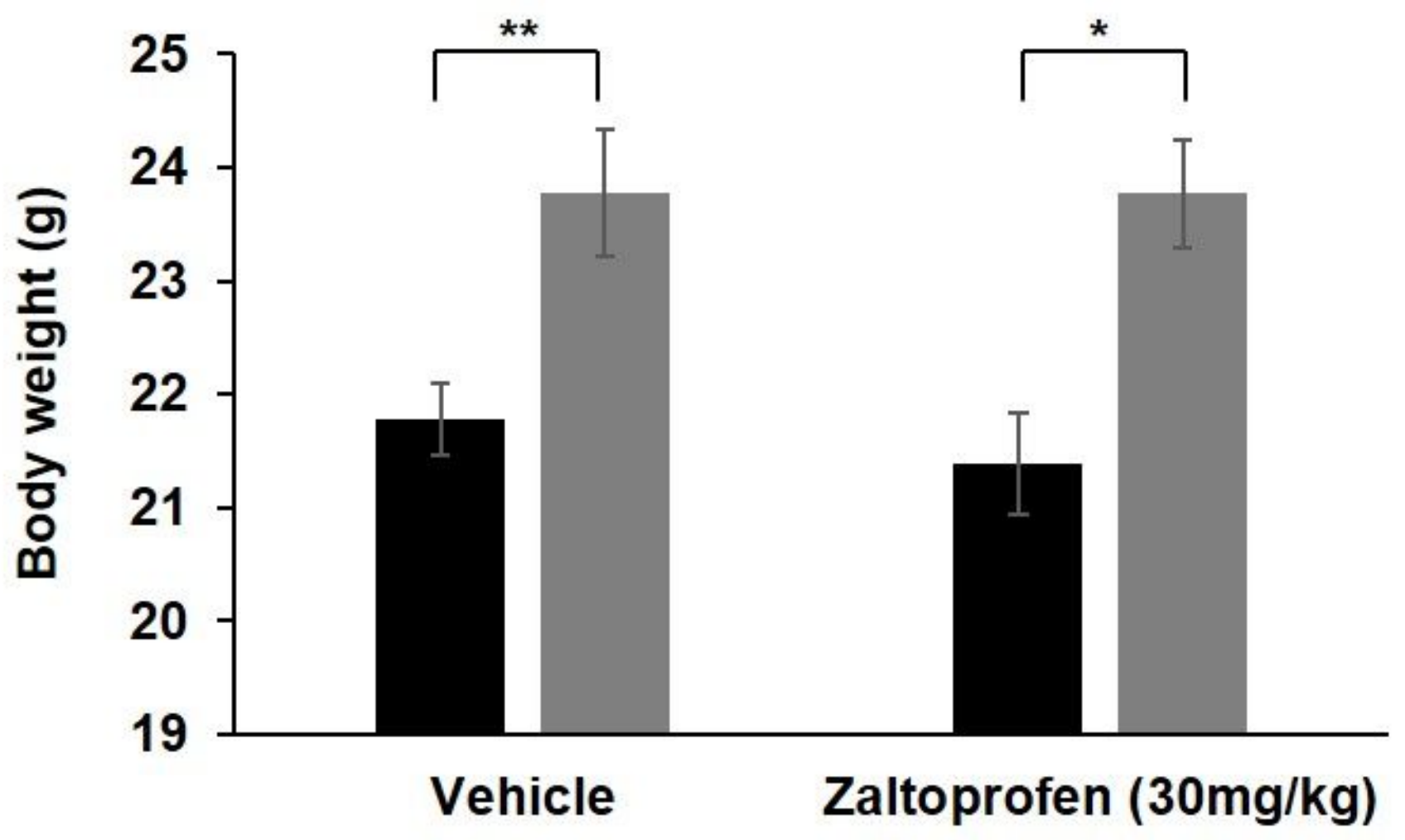

- Pre-administration $\quad$ Post-administration

Figure 7

Changes in the pre- and post-treatment body weight of mice. ${ }^{*} p<0.05,{ }^{*} \mathrm{p}<0.01$. Error bars: \pm SEM. 